UT-777

June 1997

\title{
On a Lorentz covariant matrix regularization of membrane theories
}

\author{
Kazuo Fujikawa and Kazumi Okuyama \\ Department of Physics, University of Tokyo \\ Bunkyo-ku, Tokyo 113, Japan
}

\begin{abstract}
A Lorentz covariant matrix regularization of membrane thories is studied. It is shown that the action for a bosonic membrane can be defined by matrix regularization in a Lorentz covariant manner. The generator of area preserving diffeomorphism can also be consistently defined by matrix regularization, and we can make the area preserving gauge symmetry manifest. However, the reparametrization BRST charge explicitly depends on a specific basis set introduced to define the matrix regularization. We also briefly comment on an extension of the present formulation to a supermembrane.
\end{abstract}

\section{Introduction}

The dynamics of quantum membranes is not well understood yet[1] - [14]. First of all, the world volume dynamics of membranes is not renormalizable not only for bosonic membranes but also for supermembranes. As an attempt to understand the dynamics of membranes better, a matrix regularization[8][12] received much attention recently [15] - [18]. It is hoped that the matrix regularization, if properly treated ,might lead to a non-perturbative treatment of quantized membranes.

The matrix regularization of a supermembrane in Ref.[8] is based on the light-cone gauge formulation. In fact, the covariant quantization of the supermembrane is involved , and for this reason we here mainly study a Lorentz covariant matrix regularization of bosonic membranes. A brief comment on a supermembrane is made at the end of this note. It is true that certain crucial aspects of quantized membranes can only be studied by the supermembrane, but all may not be lost by considering bosonic membranes, and some of important dynamical issues of the quantum theory of membranes may still be 
studied by examining the detailed dynamics of bosonic membranes. It is our hope that a Lorentz covariant matrix regularization of bosonic membranes may lead to a better understanding of quantum theory of membranes in general.

The starting Lagrangian of the bosonic membrane is given by

$$
\begin{aligned}
\mathcal{L}_{0} & =\frac{1}{2} \sqrt{-g}\left(1-g^{a b} \partial_{a} X^{\mu} \partial_{b} X_{\mu}\right) \\
& =-\frac{1}{2} \operatorname{det} \tilde{g}^{a b}-\frac{1}{2} \tilde{g}^{a b} \partial_{a} X^{\mu} \partial_{b} X_{\mu}
\end{aligned}
$$

where we defined

$$
\tilde{g}^{a b}=\sqrt{-g} g^{a b}
$$

and the indices $a$ and $b$ run over 0 to 2 . If one generalizes the conformal gauge in string theory to the case of the present membrane theory, one has

$$
g^{00}+\operatorname{det} g^{k l}=0, \text { and } g^{0 k}=0
$$

where $k, l=1,2$, or in an equivalent notation

$$
\tilde{g}^{00}+1=0, \text { and } \tilde{g}^{0 k}=0
$$

In this gauge, the world volume of membranes is reduced to a product of two- dimensional space $\Sigma$ times time coordinate $R, \Sigma \times R$. The Faddeev-Popov gauge fixing Lagrangian is given by

$$
\begin{aligned}
\mathcal{L}_{g} & =N_{a}\left(\tilde{g}^{0 a}+\delta^{0 a}\right) \\
& +i b_{a}\left[\partial_{b}\left(c^{b} \tilde{g}^{0 a}\right)-\tilde{g}^{b a} \partial_{b} c^{0}-\tilde{g}^{0 b} \partial_{b} c^{a}\right]
\end{aligned}
$$

where $N_{a}$ are the Lagrangian multiplier fields, and $c^{a}$ and $b_{a}$ stand for ghosts and antighosts, respectively. The total Lagrangian

$$
\mathcal{L}=\mathcal{L}_{0}+\mathcal{L}_{g}
$$

is written as $[9]$

$$
\mathcal{L}=\frac{1}{2} \partial_{0} X^{\mu} \partial_{0} X_{\mu}-\frac{1}{2} \operatorname{det} G_{k l}+i b_{0}\left(\partial_{0} c^{0}-\partial_{k} c^{k}\right)+i b_{k} \partial_{0} c^{k}
$$

with

$$
G_{k l}=\partial_{k} X^{\mu} \partial_{l} X_{\mu}+i b_{k} \partial_{l} c^{0}+i b_{l} \partial_{k} c^{0}
$$

after integration over $N_{a}$ and $\tilde{g}^{k l}$. The Lorentz covariant Lagrangian has a structure quite similar to that of the light-cone Lagrangian, though it contains up to quartic couplings of ghost fields. 


\section{Symplectic Structure}

To implement the matrix regularization, it is important to understand the symplectic structure. For this purpose, it is convenient to use the form notation. The symplectic form on the $2 \mathrm{~d}$ manifold $\Sigma$, whose canonical coordinates are $\sigma^{1}$ and $\sigma^{2}$, is defined by

$$
\omega=d \sigma^{1} \wedge d \sigma^{2}
$$

The Hamiltonian vector field $\vec{f}$ for a function $f\left(\sigma^{1}, \sigma^{2}\right)$ is defined by

$$
\vec{f}=\partial_{1} f \partial_{2}-\partial_{2} f \partial_{1}
$$

The "Poisson bracket", when we regard the coordinates $\sigma^{1}$ and $\sigma^{2}$ as two-dimensional phase space variables, is written in a number of ways

$$
\{f, g\} \equiv \mathcal{L}_{\vec{f}} g=\omega(\vec{f}, \vec{g})=(-d f, \vec{g})=\partial_{1} f \partial_{2} g-\partial_{2} f \partial_{1} g
$$

where $\mathcal{L}_{\vec{f}}$ stands for the Lie derivative generated by the Hamiltonian vector field $\vec{f}, \omega(\vec{f}, \vec{g})$ is the inner product of two Hamiltonian vector fields $\vec{f}$ and $\vec{g}$ with the two form $\omega$, and the notation $($, ) stands for a contraction of a 1-form and a vector field. The Poisson bracket in (2.3) satisfies

$$
\begin{aligned}
\int d^{2} \sigma\{f, g\} h & =\int d^{2} \sigma f\{g, h\} \\
d f \wedge d g & =\{f, g\} \omega
\end{aligned}
$$

In our analysis, it is convenient to regard the space components of $b_{a}, c^{a}$ as 1 -form and vector field on $\Sigma$, respectively

$$
\begin{aligned}
& \mathbf{b}=b_{1} d \sigma^{1}+b_{2} d \sigma^{2} \\
& \mathbf{c}=c^{1} \partial_{1}+c^{2} \partial_{2}
\end{aligned}
$$

Our Lorentz covariant Lagrangian is then written as

$$
\mathcal{L}=\frac{1}{2} \partial_{0} X^{\mu} \partial_{0} X_{\mu}-\frac{1}{2} \operatorname{det} G_{k l}+i b_{0}\left(\partial_{0} c^{0}-\operatorname{div} \mathbf{c}\right)+i\left(\mathbf{b}, \partial_{0} \mathbf{c}\right)
$$

with

$$
\operatorname{det} G_{k l}=\sum_{\mu<\nu}\left\{X^{\mu}, X^{\nu}\right\}\left\{X_{\mu}, X_{\nu}\right\}+2 i\left(\mathbf{b}, \vec{X}_{\mu}\right)\left\{X^{\mu}, c^{0}\right\}-3\left(\mathbf{b}, \overrightarrow{c^{0}}\right)^{2}
$$

where $\vec{X}_{\mu}$ and $\vec{c}^{0}$ are Hamiltonian vector fields associated with $X_{\mu}$ and $c^{0}$. The Lie derivatives of vector field $v$ and 1-form $\alpha$ are respectively given by $\left(\mathcal{L}_{u} v\right)^{k}=u^{l} \partial_{l} v^{k}-\partial_{l} u^{k} v^{l}$ and $\left(\mathcal{L}_{u} \alpha\right)_{k}=u^{l} \partial_{l} \alpha_{k}+\partial_{k} u^{l} \alpha_{l}$. 
If one uses a gauge $\tilde{g}^{00}=-\rho\left(\sigma_{1}, \sigma_{2}\right)$ instead of (1.4), the Lagrangian (1.7) is written as

$$
\rho^{-1} \mathcal{L}=\frac{1}{2} \partial_{0} X^{\mu} \partial_{0} X_{\mu}-\frac{1}{2} \operatorname{det} G_{k l}+i b_{0}\left(\partial_{0} c^{0}-\rho^{-1} \partial_{k}\left(\rho c^{k}\right)\right)+i b_{k} \partial_{0} c^{k}
$$

and the symplectic form becomes $\omega=\rho d \sigma^{1} \wedge d \sigma^{2}$. The Poisson bracket (2.3) is then replaced by

$$
\{f, g\}=\rho^{-1}\left(\partial_{1} f \partial_{2} g-\partial_{2} f \partial_{1} g\right)
$$

Also, divc $=\rho^{-1} \partial_{k}\left(\rho c^{k}\right)$ and $V=\rho^{-1}\left(\partial_{1} b_{2}-\partial_{2} b_{1}\right)=* d \mathbf{b}$ in (3.9) below. The $\rho$ plays a role of density [8]. In this paper we work with the gauge (1.4).

\section{Canonical Formalism and Symmetry Properties}

The Hamiltonian corresponding to our covariant Lagrangian is given by

$$
\mathcal{H}=\frac{1}{2} P^{\mu} P_{\mu}+\frac{1}{2} \operatorname{det} G+i b_{0} \partial_{k} c^{k}
$$

with the basic canonical commutation relations

$$
\begin{aligned}
& {\left[X_{\mu}(\sigma), P_{\nu}\left(\sigma^{\prime}\right)\right]=i \eta_{\mu \nu} \delta^{(2)}\left(\sigma-\sigma^{\prime}\right)} \\
& {\left[b_{a}(\sigma), c^{b}\left(\sigma^{\prime}\right)\right]_{+}=\delta_{a}^{b} \delta^{(2)}\left(\sigma-\sigma^{\prime}\right)}
\end{aligned}
$$

where $P_{\mu}(\sigma)=\partial_{0} X_{\mu}(\sigma)$, and $[,]_{+}$stands for an anti-commutator of operators.

We now analyze the equations of motion. We start with the equations of motion for $c^{0}$ and $b_{k}$

$$
\begin{aligned}
& \partial_{0} c^{0}-\operatorname{divc}=0 \\
& \partial_{0} b_{k}-\partial_{k} b_{0}=0
\end{aligned}
$$

where $k=1,2$, and the 1 st equation corresponds to the conservation of "current" $c^{a}$, and the 2 nd equation shows that the $0 k$-components of $d b_{a}$ (in a 3-dimensional notation) vanishes. The equations of motion for $\mathbf{c}$ and $b_{0}$ are given by

$$
\begin{aligned}
\partial_{0} \mathbf{c} & =\vec{X}_{\mu}\left\{X^{\mu}, c^{0}\right\}+3 i \overrightarrow{c^{0}}\left(\mathbf{b}, \overrightarrow{c^{0}}\right) \\
\partial_{0} b_{0} & =-\left\{\left(\mathbf{b}, \overrightarrow{X_{\mu}}\right), X^{\mu}\right\}-3 i *\left(\mathcal{L}_{c^{0}} \mathbf{b} \wedge \mathbf{b}\right)
\end{aligned}
$$

where the star $(\star)$ operation transforms a two form to its dual (i.e., scalar in the present case). The constant shift in $b_{0}$, which is a symmetry of our action, is generated by

$$
\begin{aligned}
b_{0} & \rightarrow b_{0}+\xi \\
\text { charge } & =\int d^{2} \sigma c^{0}
\end{aligned}
$$


Our Lagrangian is also invariant under an addition of a (time independent) hamiltonian vector field $\vec{w}$ to $\mathbf{c}$, and this symmetry is generated by the operator $V$ defined by

$$
\begin{aligned}
\mathbf{c} & \rightarrow \mathbf{c}+\vec{w} \\
V & =\partial_{1} b_{2}-\partial_{2} b_{1}=* d \mathbf{b}
\end{aligned}
$$

In fact, the ghost part of the action (2.6) is invariant under $\mathbf{c} \rightarrow \mathbf{c}+\mathbf{f}$ with a more general(time independent) vector field $\mathbf{f}$ with divf $=0$. The basic BRST transformation in our covariant formulation is defined by

$$
\begin{aligned}
\delta_{B R S T} X^{\mu} & =\epsilon c^{a} \partial_{a} X^{\mu} \\
\delta_{B R S T} c^{a} & =\epsilon c^{b} \partial_{b} c^{a} \\
\delta_{B R S T} b_{a} & =i \epsilon B_{a}
\end{aligned}
$$

where $\epsilon$ is a Grassmann parameter, and

$$
\begin{gathered}
B_{0}=\frac{1}{2}\left(\partial_{0} X^{\mu}\right)^{2}+\frac{1}{2} \operatorname{det} G+i \partial_{0} b_{0} c^{0}+i \partial_{k} b_{0} c^{k}+2 i b_{0} \partial_{0} c^{0}+i b_{k} \partial_{0} c^{k} \\
B_{k}=\partial_{0} X^{\mu} \partial_{k} X_{\mu}+i \partial_{0}\left(b_{k} c^{0}\right)+i \partial_{l} b_{k} c^{l}+i b_{l} \partial_{k} c^{l}+2 i b_{0} \partial_{k} c^{0}
\end{gathered}
$$

These variables $B_{a}$, up tp equations of motion, correspond to the components $T_{0 a}$ of the energy momentum tensor on the world volume. The BRST charge is defined by[9]

$$
\begin{aligned}
Q= & \int d^{2} \sigma\left[c^{0}\left(\frac{1}{2}\left(\partial_{0} X^{\mu}\right)^{2}+\frac{1}{2} \operatorname{det} G\right)\right. \\
& \left.+c^{k} \partial_{0} X^{\mu} \partial_{k} X_{\mu}-i b_{0}\left(c^{0} \partial_{k} c^{k}+c^{k} \partial_{k} c^{0}\right)-i b_{k} c^{l} \partial_{l} c^{k}\right]
\end{aligned}
$$

By using the BRST charge and the operator $V$, the generator $L$ of the area preserving diffeomorphism is given by [13]

$$
L=[Q, V]_{+}=\partial_{1} B_{2}-\partial_{2} B_{1}=* d \mathbf{B}
$$

and

$$
L_{w}=\int d^{2} \sigma w L=\int d^{2} \sigma(-\mathbf{B}, \vec{w})
$$

We here defined

$$
\begin{aligned}
\mathbf{B} & =\partial_{0} X^{\mu} d X_{\mu}+i \partial_{0}\left(\mathbf{b} c^{0}\right)+2 i b_{0} d c^{0}-i \mathcal{L}_{\mathbf{c}} \mathbf{b} \\
L & =\left\{\partial_{0} X^{\mu}, X_{\mu}\right\}+2 i\left\{b_{0}, c^{0}\right\}+i \partial_{0}\left(\mathbf{b}, \overrightarrow{c^{0}}\right)+i \partial_{0}\left(V c^{0}\right)+i V c+i(d V, \mathbf{c})
\end{aligned}
$$


with $c=$ divc. If we use the equations of motion for ghost variables, we can rewrite $\mathbf{B}$ and $L$ as

$$
\begin{aligned}
\mathbf{B} & =\partial_{0} X^{\mu} d X_{\mu}+i b_{0} d c^{0}+i d\left(b_{0} c^{0}\right)+i \mathbf{b} c-i \mathcal{L}_{\mathbf{c}} \mathbf{b} \\
L & =\left\{\partial_{0} X^{\mu}, X_{\mu}\right\}+i\left\{b_{0}, c^{0}\right\}+i(\mathbf{b}, \vec{c})+2 i V c+i(d V, \mathbf{c})
\end{aligned}
$$

The transformation laws of area preserving diffeomorphism are given as

$$
\begin{aligned}
-i\left[L_{w}, X\right] & =\{w, X\} \\
-i\left[L_{w}, c^{0}\right] & =\left\{w, c^{0}\right\} \\
-i\left[L_{w}, b^{0}\right] & =\left\{w, b^{0}\right\} \\
-i\left[L_{w}, c^{1}\right] & =\left\{w, c^{1}\right\}-\mathcal{L}_{\mathbf{c}}(\vec{w})^{1}=\left(\mathcal{L}_{\vec{w}} \mathbf{c}\right)^{1} \\
-i\left[L_{w}, c^{2}\right] & =\left\{w, c^{2}\right\}-\mathcal{L}_{\mathbf{c}}(\vec{w})^{2}=\left(\mathcal{L}_{\vec{w}} \mathbf{c}\right)^{2} \\
-i\left[L_{w}, b_{1}\right] & =\left\{w, b_{1}\right\}+\left(\mathbf{b}, \partial_{1} \vec{w}\right)=\left(\mathcal{L}_{\vec{w}} \mathbf{b}\right)_{1} \\
-i\left[L_{w}, b_{2}\right] & =\left\{w, b_{2}\right\}+\left(\mathbf{b}, \partial_{2} \vec{w}\right)=\left(\mathcal{L}_{\vec{w}} \mathbf{b}\right)_{2}
\end{aligned}
$$

In these equations, $[$,$] in the left-hand side is a commutator of operators and \{$,$\} in the$ right-hand side is a Poisson bracket. In general, the transformation law of an operator $\mathcal{O}$ is written as

$$
i\left[L_{w}, \mathcal{O}\right]=-\mathcal{L}_{\vec{w}} \mathcal{O}
$$

with $\vec{w}$ a Hamiltonian vector field associated with $w$.

We here note interesting algebraic relations satisfied by the symmetry generators $V_{\xi}=$ $\int d^{2} \sigma \xi V$ and $L_{w}=\int d^{2} \sigma w L$ in our Lagrangian

$$
\begin{aligned}
{\left[V_{\xi}, V_{\eta}\right] } & =0 \\
{\left[L_{w}, V_{\xi}\right] } & =i V_{\{w, \xi\}} \\
{\left[L_{f}, L_{g}\right] } & =i L_{\{f, g\}}
\end{aligned}
$$

where $V$ is defined in $(3.9)$.

In connection with the area preserving gauge symmetry, we note that the action may be defined anew by using the Hamiltonian in (3.1) as

$$
\mathcal{L}=P^{\mu} \partial_{0} X_{\mu}+i b_{a} \partial_{0} c^{a}-\mathcal{H}+\delta_{B R S T}(i A V)
$$

where we added a BRST exact term to the action, which does not change the physical sector of the Fock space,

$$
\begin{aligned}
\int d^{2} \sigma \delta_{B R S T}(i A V) & =\int d^{2} \sigma(i \lambda V-A L) \\
& =\int d^{2} \sigma[i(\mathbf{b}, \vec{\lambda})+(\mathbf{B}, \vec{A})]
\end{aligned}
$$


The transformation properties of the auxiliary fields $A$ and $\lambda$ under the BRST transformation, the symmetry genrated by $V$ and the area preserving diffeomorphism generated by $L$ are respectively defined by

$$
\begin{array}{ll}
\delta_{B R S T} A=\lambda, & \delta_{B R S T} \lambda=0 \\
\delta_{V} \mathbf{c}=\vec{\xi}, & \delta_{V} \lambda=D_{0} \xi \\
\delta_{L} A=D_{0} w, & \delta_{L} \lambda=-\{w, \lambda\}
\end{array}
$$

and for other generic variables

$$
\delta_{L} \mathcal{O}=-\mathcal{L}_{\vec{w}} \mathcal{O}
$$

where we defined $D_{0} f=\partial_{0} f+\{A, f\}$. The significance of two auxiliary variables $A$ and $\lambda$ becomes transparent when one integrates over $P_{\mu}$ in the above Lagrangian (3.21). We then obtain

$$
\mathcal{L}=\frac{1}{2}\left(D_{0} X^{\mu}\right)^{2}-\frac{1}{2} \operatorname{det} G_{k l}+i b_{0}\left(D_{0} c^{0}-\operatorname{div} \mathbf{c}\right)+i\left(\mathbf{b}, D_{0} \mathbf{c}+\vec{\lambda}\right)
$$

where we defined

$$
D_{0} \mathcal{O}=\partial_{0} \mathcal{O}+\mathcal{L}_{\vec{A}} \mathcal{O}
$$

for a general operator $\mathcal{O}$. By this way, we can make the area preserving gauge symmetry explicit in (3.25), and the auxiliary variable $A$ corresponds to the gauge field for this symmetry and $\lambda$ is a BRST partner of $A ; \lambda$ can be regarded as a gauge field for the symmetry generated by $V$, as is expected from the first expression in (3.22). In this formulation, our original covariant Lagrangian corresponds to the gauge fixing $A=\lambda=0$ of area preserving gauge symmetry:This is realized by adding the gauge fixing terms $N A-i \xi \lambda$ to (3.25) with a Nakanishi-Lautrup doublet $(\xi, N)$. See also Ref.[8] for a related analysis in the light-cone gauge. In the following we work with our original covariant Lagrnagian (2.6).

\section{Solving Constraints and Matrix Regularization}

We have constraints on the physical states in our formulation

$$
\begin{aligned}
& V \mid \text { phys }\rangle=d \mathbf{b} \mid \text { phys }\rangle=0 \\
& L \mid \text { phys }\rangle=d \mathbf{B} \mid \text { phys }\rangle=0
\end{aligned}
$$

in addition to the BRST symmetry $Q|p h y s\rangle=0$. We solve the first constraint (4.1) in the operator level by writing $\mathbf{b}$ in a locally exact form

$$
\mathbf{b}=-d b
$$


and thus $V \simeq 0$ in the sense of Dirac in (3.14). At the same time, we introduce a new variable defined by

$$
c \equiv \operatorname{divc}
$$

Note that $c$ is invariant under the transformation generated by $V$. The operations in (4.3) and (4.4) are regarded as a gauge fixing of $V$-symmetry by using the gauge condition $F=\partial_{1} c^{2}-\partial_{2} c^{1}=0$. This is seen by the following path integral manipulation

$$
\begin{aligned}
& \int \mathcal{D} \mathbf{b} \mathcal{D} \mathbf{c} \delta(V) \delta(F) \operatorname{det}\{V, F\}^{-1} \exp (i S) \\
= & \int \mathcal{D} \mathbf{b} \mathcal{D} \mathbf{c} \delta(V) \delta(F) \operatorname{det} \triangle^{-1} \exp (i S) \\
= & \int \mathcal{D} b \mathcal{D} c \exp \left(i S^{\prime}\right)
\end{aligned}
$$

The Lagrangian (2.6) is then written as

$$
\mathcal{L}^{\prime}=\frac{1}{2} \partial_{0} X^{\mu} \partial_{0} X_{\mu}-\frac{1}{2} \operatorname{det} G^{\prime}+i b_{0}\left(\partial_{0} c^{0}-c\right)+i b \partial_{0} c
$$

with

$$
\operatorname{det} G^{\prime}=\sum_{\mu<\nu}\left\{X^{\mu}, X^{\nu}\right\}\left\{X_{\mu}, X_{\nu}\right\}+2 i\left\{b, X_{\mu}\right\}\left\{X^{\mu}, c^{0}\right\}-3\left\{b, c^{0}\right\}^{2}
$$

All the variables here are treated as functions on $\Sigma$, and the variables $b$ and $c$ become canonical conjugate to each other. If we use the equations of motion for ghost variables

$$
\begin{aligned}
& \partial_{0} c^{0}-c=0 \\
& \partial_{0} b+b_{0}=0
\end{aligned}
$$

the generator of the area preserving diffeomorphism in (3.17) is further rewritten as

$$
\begin{aligned}
\left.L\right|_{\mathbf{b}=-d b} & =\left\{\partial_{0} X^{\mu}, X_{\mu}\right\}+2 i\left\{b_{0}, c^{0}\right\}+i \partial_{0}\left\{b, c^{0}\right\} \\
& =\left\{\partial_{0} X^{\mu}, X_{\mu}\right\}+i\left\{b_{0}, c^{0}\right\}+i\{b, c\} \equiv L^{\prime}
\end{aligned}
$$

The transformation law of general variable $\mathcal{O}$ appearing in (4.6) under the area preserving diffeomorphism is written as

$$
\delta \mathcal{O}=\{w, \mathcal{O}\}
$$

with the generator of this transformation being given by $L^{\prime}(4.9)$.

We solved the constraint $V=0$ in the operator level, but its BRST transform $L^{\prime}=0$ is not solved in the operator level in our treatment. The BRST symmetry, which is manifest in (4.1) and (4.2), is no more manifest after solving $V=0$. The gauge symmetry generated by $V$ (and also by $L$ ) is characterized by a time independent parameter $\vec{w}$, and in this sense it is non-dynamical and analogous to the residual symmetry of $A_{0}=0$ gauge 
for Yang-Mills fields. This symmetry plays an important role to eliminate non-oscillatory (instability) modes when one compactifies two spatial coordinates in membrane theory[13].

In passing, the "Poisson bracket" of two fermionic( Grassmann) variables $\xi$ and $\eta$ are defined by

$$
\{\xi, \eta\} \equiv \partial_{1} \xi \partial_{2} \eta-\partial_{2} \xi \partial_{1} \eta
$$

One can then confirm

$$
\{\xi, \eta\}=\{\eta, \xi\}
$$

by noting the anti-commuting property of $\xi$ and $\eta$. In the matrix regularization, the fermionic variables are then specified by the anti-commutator of two matrix valued fermionic variables.

The Lagrangian (4.6) and the generator of the area preserving diffeomorphism (4.9) are both written solely in terms of Poisson brackets or bi-linear combinations of two field variables. It is thus straightforward to introduce the matrix regularization by formally replacing the Poisson bracket by an (anti-)commutator of matrix valued operators. This transition is facilitated by expanding the various operators in terms of a complete set of basis vectors defined on the two-dimensional space $\Sigma$.

For example,

$$
\begin{aligned}
X^{\mu}\left(\tau, \sigma_{1}, \sigma_{2}\right) & \equiv \sum_{A} X_{A}^{\mu}(\tau) Y^{A}\left(\sigma_{1}, \sigma_{2}\right) \\
b\left(\tau, \sigma_{1}, \sigma_{2}\right) & \equiv \sum_{A} b_{A}(\tau) Y^{A}\left(\sigma_{1}, \sigma_{2}\right)
\end{aligned}
$$

and similarly for other variables with a suitable complete orthonormal set of functions $\left\{Y^{A}\left(\sigma_{1}, \sigma_{2}\right)\right\}$ on the space $\Sigma$. We then formally replace the set $\left\{Y^{A}\left(\sigma_{1}, \sigma_{2}\right)\right\}$ by the (hermitian) generators of the group $S U(N)$ with $N \rightarrow \infty$ [8]. The dynamical varibles are then promoted to (infinite dimensional) matrices and we have, for example,

$$
\begin{aligned}
\int d^{2} \sigma \partial_{0} X^{\mu} \partial_{0} X_{\mu}= & \operatorname{Tr} \partial_{0} X^{\mu} \partial_{0} X_{\mu} \\
\int d^{2} \sigma \operatorname{det} G^{\prime}= & \operatorname{Tr}\left(-\sum_{\mu<\nu}\left[X^{\mu}, X^{\nu}\right]\left[X_{\mu}, X_{\nu}\right]\right. \\
& \left.-2 i\left[b, X_{\mu}\right]\left[X^{\mu}, c^{0}\right]+3\left[b, c^{0}\right]_{+}\left[b, c^{0}\right]_{+}\right)
\end{aligned}
$$

where $\operatorname{Tr}$ specifies the trace operation, and [ , ] stands for the commutator and [ , ]+ for the anti-commutator. In the matrix notation, we can write the action, the Hamiltonian and the generator of the area preserving diffeomorphism respectively as

$$
\begin{aligned}
\int d \tau d^{2} \sigma \mathcal{L} & =\int d \tau \operatorname{Tr}\left(\frac{1}{2} \partial_{0} X^{\mu} \partial_{0} X_{\mu}-\frac{1}{2} \operatorname{det} G^{\prime}+i b_{0}\left(\partial_{0} c^{0}-c\right)+i b \partial_{0} c\right) \\
H & =\int d^{2} \sigma \mathcal{H}=\operatorname{Tr}\left(\frac{1}{2} P^{\mu} P_{\mu}+\frac{1}{2} \operatorname{det} G^{\prime}+i b_{0} c\right) \\
\int d^{2} \sigma w(\sigma) L^{\prime} & =\operatorname{Tr} w\left(-i\left[\partial_{0} X^{\mu}, X_{\mu}\right]+\left[b_{0}, c^{0}\right]_{+}+[b, c]_{+}\right)
\end{aligned}
$$


where $P^{\mu}=\partial_{0} X^{\mu}$ and we make the replacement (4.14) inside $\operatorname{det} G^{\prime}$. The parameter $w(\sigma)$ is also expanded in terms of the basis set $\left\{Y^{A}\left(\sigma_{1}, \sigma_{2}\right)\right\}$.

\section{Discussion}

We have shown that the action and the generator of area preserving diffeomorphism for bosonic membranes can be consistently defined by Lorentz covariant matrix regularization, if one keeps $N$ of $S U(N)$ in (4.15) finite. Our matrix regularized Lagrangian has a structure very similar to that of the light-cone gauge[8]. This suggests that the basic dynamics of membrane theory is not much influenced by the infinite momentum frame which is the underlying physical picture of the light-cone gauge. However, it is important to recognize that the BRST charge (3.13) in our formulation cannot be written in a simple matrix notation by using commutators for bosonic variables and anti-commutators for fermionic variables even in the formal way. For example, we cannot make the replacement in (4.4) consistently, and the variables $c^{k}$ survive in the BRST charge till the end. This means that one cannot eliminate the explicit dependence on a particular basis set $\left\{Y^{A}\left(\sigma_{1}, \sigma_{2}\right)\right\}$ in the Lorentz covariant formulation, while the basis set $\left\{Y^{A}\left(\sigma_{1}, \sigma_{2}\right)\right\}$ carries the information of the topology of world volume.

Our analysis suggests that Lorentz covariance and unitarity ( or BRST symmetry), both of which are essential for any sensible theory, cannot be simultaneously maintained in matrix regularization in a manner independent of world volume topology. Although it is premature to make a definite statement at this moment, our consideration at least appears to be consistent with the recent analysis by Matsuo et al. [15]. They examined the Lorentz covarince of membrane theory in the (manifestly unitary) light-cone gauge formulation, and they conclude that the explicit appearance of the world volume metric, which contains the information of topology, is required to recover the full Lorentz covariance.

Ultimately, one may want to analyze the dynamics of supermembrane in a Lorentz covariant manner not only in continuum notation but also in matrix regularization. The 32-component spinor $\theta\left(\tau, \sigma_{1}, \sigma_{2}\right)$ in $d=11$ dimensional space is irreducible, and any algebraic gauge fixing of the $\kappa$-symmetry associated with $\theta\left(\tau, \sigma_{1}, \sigma_{2}\right)$ generally breaks the Lorentz symmetry $S O(10,1)$. One possible algebraic gauge fixing of $\kappa$ symmetry is to decompose

$$
\theta\left(\tau, \sigma_{1}, \sigma_{2}\right)=\theta_{L}+\theta_{R}
$$

and to impose the gauge condition $\theta_{R}=0[19,20,21$. This breaks the symmetry $S O(10,1)$ down to $S O(9,1)$. If one combines this algebraic gauge fixing with the $S O(10,1)$ covariant treatment of reparametrization symmetry in Ref.[9] one can define an $S O(9,1)$ invariant supermembrane theory consisting of polynomials up to quartic terms. The theory thus 
defined is located in between the full covariant treatment and the light-cone gauge. This formulation may be useful in the analysis of dynamical issues related to M-theory 16, 17, 18], for example. The supersymmetry algebra in this formulation exhibits interesting properties, and a detailed account of this analysis will be reported elsewhere.

\section{References}

[1] P.A.M. Dirac, Proc. R. Soc. A268 (1962)57.

[2] P.A. Collins and P.W. Tucker,Nucl.Phys. B112 (1976)150.

P.S. Howe and P.W. Tucker, J.Phys.A10 (1977)L155.

[3] A. Sugamoto, Nucl. Phys. B215 (1983)381.

[4] M. Henneaux, Phys. Lett. B120 (1983)179.

[5] K. Kikkawa and M. Yamasaki, Prog. Theor. Phys. 76 (1986)1379.

[6] E. Bergshoeff, E. Sezgin and P.K. Townsend, Phys. Lett. B189 (1987)75.

[7] M.J. Duff, P.S. Howe, T. Inami and K.S. Stelle, Phys. Lett. B191 (1987)70.

[8] B. de Wit, J. Hoppe and H. Nicolai, Nucl. Phys. B305 (1988)545.

[9] K. Fujikawa and J. Kubo, Phys. Lett. B199 (1987)75.

[10] M.J. Duff, T. Inami, C.N. Pope, E. Sezgin and K.S. Stelle, Nucl. Phys. B297 (1988)515.

[11] L. Mezincescu, R.I. Nepomechie and P. van Nieuwenhuizen, Nucl. Phys. B309 (1988)317.

[12] B. de Wit, M. Luescher and H. Nicolai, Nucl. Phys. B320 (1989)135.

[13] K. Fujikawa and J. Kubo, Nucl. Phys. B356 (1991)208.

[14] For a review of early works of membrane theory, E. Bergshoeff, E. Sezgin and P.K. Townsend, Ann. Phys. 185 (1988)330.

[15] K. Ezawa, Y. Matsuo and K. Murakami, hep-th/9705005.

[16] For a review of membrane theory in the context of M-theory, M.J. Duff, hep-th/9611203. 
[17] T. Banks, W. Fischler, S.H. Shenker and L. Susskind, Phys.Rev.D55 (1997) 5112, hep-th/9610043.

[18] P.K. Townsend, Phys.Lett. B373 (1996) 68, hep-th/9512062.

[19] R. Kallosh, hep-th/9705056.

[20] E. Bergshoeff, R. Kallosh, T. Ortin and G. Papadopoulos, hep-th/975040.

[21] M. Aganagic, C. Popescu and J.H. Schwarz, Phys.Lett.B393 (1997)311 hepth/9610249.

M. Aganagic, C. Popescu and J.H. Schwarz, hep-th/9612080. 